\title{
ELECTRONIC CONTROL OF MOLECULAR CONFIGURATION INSTABILITY VIA VIBRONIC COUPLING. PSEUDO JAHN-TELLER STABILIZATION OF VERTICALLY EXCITED STATES OF $\mathrm{F}_{2} \mathrm{CO}, \mathrm{N}_{2} \mathrm{H}_{2}$ AND $\mathrm{H}_{2} \mathrm{C}_{2} \mathrm{O}$ MOLECULES
}

\author{
Natalia Gorinchoy \\ Institute of Chemistry of Academy of Sciences of Moldova, 3, Academiei str., Chisinau MD-2028, Republic of Moldova \\ e-mail:ngorinchoy@yahoo.com
}

\begin{abstract}
The pseudo Jahn-Teller effect is employed to explain the origin of distortions of carbonyl fluoride, diazene and ketene molecules in the lowest singlet and triplet excited states. The ground state and the excited electronic states of considered molecules were calculated by ab initio SCF CI method with the use of the 631G-type basis set. The corresponding pseudo Jahn-Teller coupling constants are estimated by fitting the $a b$ initio data for the adiabatic potential curves of the molecules to the general formula, obtained from the vibronic theory. With these data an explanation of both the larger instability of the triplet excited states as compared with the singlet ones and the elongation of the corresponding bond distances in the excited states is obtained.
\end{abstract}

Keywords: Pseudo Jahn-Teller effect, stereochemistry, excited states, carbonyl fluoride, diazene, ketene.

\section{Introduction}

The stereochemistry of molecular systems is determined by their electronic structure and the permutation symmetry of identical atoms. The latter means that from the viewpoint of symmetry any molecule must possess the spatial structure with the highest possible symmetry. However, depending on the electronic state the high-symmetry nuclear configuration can become unstable, and the molecule is distorted. Any perturbation of molecular system (excitation, ionization, coordination to transition metal complexes etc.) changes its electronic state and, as a consequence, may lead to distortion of its nuclear configuration.

The problem of electronic control on nuclear configuration instability has already some history. In the case of degenerate electronic states the configuration instability is a direct consequence of the Jahn-Teller effect (See, e.g. in [13]). It has also been shown that the only reason of instability of the high-symmetry nuclear configurations and structural distortions of any molecular system in the non-degenerate state is the pseudo Jahn-Teller effect (PJTE), that is the vibronic mixing of considered state with the appropriate by symmetry excited states [1, 2, 4-7]. Hence the instabilities should be sought for in the electronic states which mix strongly under nuclear displacements, and vice versa. If there is instability, there should be corresponding excited states that cause the instability of the reference configuration. The electronic control of the instabilities via vibronic coupling has been considered in detail in a series of papers [4-13].

One of the examples of electronic control on nuclear configuration instability is the stabilization of vertically excited states. The available experimental and theoretical data indicate that in the electronically excited states molecules may adopt equilibrium geometries very different from those in the ground states. Moreover, the distortions of some molecules in the lowest triplet state are stronger than in the excited singlet state of the same symmetry. In [14] the pseudo Jahn-Teller effect was used to explain the origin of the carbonyl group bending in the lowest excited states of formaldehyde molecule. It was shown that the larger distortion of formaldehyde in the triplet excited state as compared with the singlet one can be explained by the larger vibronic contribution to the curvature of the adiabatic potential (AP) for the triplet state. However, the non-vibronic contribution to the curvature of the AP has not been evaluated, and the resulting values of the curvature along the distortion coordinate in these two states were not obtained.

In the present work the pseudo Jahn-Teller effect is employed to reveal the origin of geometry peculiarities of carbonyl fluoride $\mathrm{F}_{2} \mathrm{CO}$, diazene $\mathrm{N}_{2} \mathrm{H}_{2}$ and ketene $\mathrm{H}_{2} \mathrm{C}_{2} \mathrm{O}$ molecules in the excited states. These molecules are rather well studied. All of them are planar in the ground electronic states, but have distorted geometry of lower symmetry in the excited states. Thus, for the tetraatomic carbonyl molecules $\mathrm{X}_{2} \mathrm{CO}(\mathrm{X}=\mathrm{H}, \mathrm{F}, \mathrm{Cl})$ it was shown experimentally and by quantum chemical calculations that electronic excitation from the ground to the lowest excited singlet and triplet states results in lengthening of C-O distance and pyramidalization of the carbonyl fragment, which is planar in the ground state (See, for example [15-17] and references herein). Moreover, in the triplet excited state distortion is stronger than in the singlet one (Figures $1 \mathrm{~b}, 1 \mathrm{c}$ ).

The diimide molecule $\mathrm{N}_{2} \mathrm{H}_{2}$ (also referred to as 1,2-diazene) has a trans-planar shape of $\mathrm{C}_{2 h}$ symmetry in the ground ${ }^{~} \mathrm{~A}_{\mathrm{g}}$ state, but the lowest excited singlet and triplet states are predicted to have nonplanar "skewed" anticline $\left(\mathrm{C}_{2}\right)$ structures, the dihedral angle being by $\approx 10^{\circ}$ smaller for the triplet state compared with the singlet one (Figure 2) [18-20]. 


$$
\angle \boldsymbol{\alpha}_{\mathrm{T}}>\angle \boldsymbol{\alpha}_{\mathrm{S}}
$$

a)

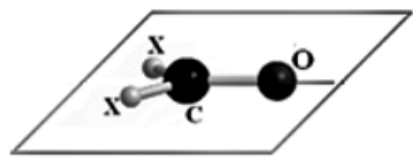

Ground state ${ }^{1} \mathrm{~A}$ $\left(\mathrm{C}_{2 \mathrm{v}}\right.$ symmetry)

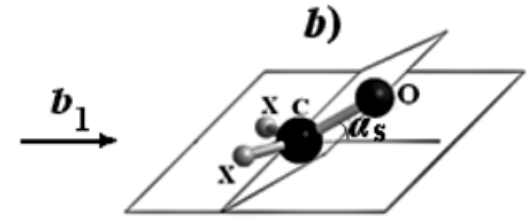

Excited singlet state ${ }^{1} \mathrm{~A}$ " $\left({ }^{\mathbf{1}} \mathbf{A}_{\mathbf{2}}\right)$ ( $\mathrm{C}_{\mathrm{s}}$ symmetry)

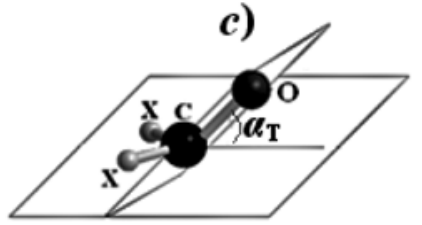

Excited triplet state ${ }^{3} \mathrm{~A} ",\left({ }^{3} \mathbf{A}_{2}\right)$ ( $\mathrm{C}_{\mathrm{s}}$ symmetry)

Figure 1. Geometry of $\mathrm{X}_{2} \mathrm{CO}$ molecules in (a) the ground state ${ }^{1} \mathrm{~A}_{1},(b)$ the excited singlet state ${ }^{1} \mathrm{~A}_{2}$, and (c) the excited triplet state ${ }^{3} \mathrm{~A}_{2}$.

$$
\angle \boldsymbol{\alpha}_{\mathrm{T}}<\angle \boldsymbol{\alpha}_{\mathrm{S}}
$$
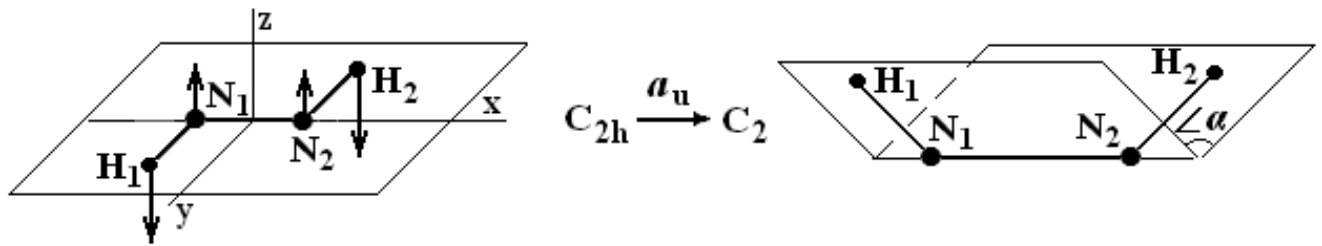

Figure 2. Geometry of $\mathrm{N}_{2} \mathrm{H}_{2}$ molecule in the ground ${ }^{1} \mathrm{~A}_{\mathrm{g}}$ and excited ${ }^{1,3} \mathrm{~B}_{\mathrm{g}}$ state singlet states.

The arrows show the displacement of $a_{\mathrm{u}}$ type leading to the nonplanar $\mathrm{C}_{2}$ structure.

Ketene molecule in the ground ${ }^{1} \mathrm{~A}_{1}$ electronic state is also planar with the nuclear configuration of $\mathrm{C}_{2 \mathrm{v}}$ symmetry, while it has peculiar geometries in the excited states. It was established that in the first excited triplet state the molecule undergoes the in-plane distortion leading to the in-plane $\mathrm{C}_{\mathrm{S}}$ structure $\left(\mathrm{C}_{\mathrm{S}}\right.$ " in Figure 3) with the elongated $\mathrm{C}$ - $\mathrm{C}$ bond, while in the second triplet state it has the out-of-plane distorted nuclear configuration $\left(\mathrm{C}_{\mathrm{S}}{ }^{\perp}\right.$ in Figure 3$)$, also with the elongated $\mathrm{C}-\mathrm{C}$ bond $[21,22]$.
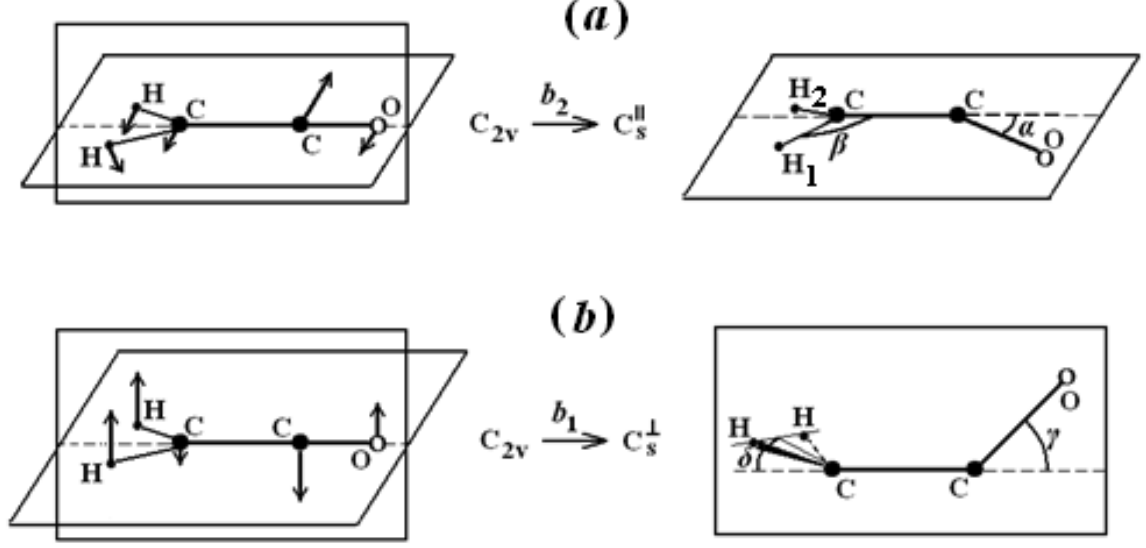

(b)
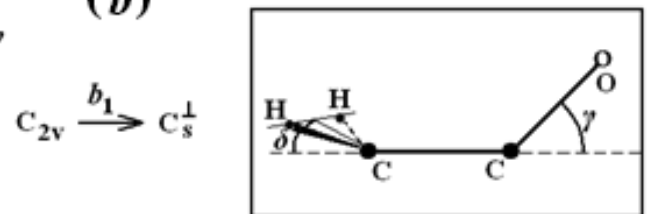

Figure 3. Ketene geometries in the ground $\left(C_{2 v}\right)$ and low-lying ${ }^{3} A_{2}\left(C_{s}{ }^{I I}\right)$ and ${ }^{3} A_{1}\left(C_{s}^{\perp}\right)$ excited states.

In what is designated $\mathrm{C}_{\mathrm{S}}{ }^{\mathrm{II}}$, the molecule is planar but the oxygen and the $\mathrm{CH}_{2}$ group are bent from the $\mathrm{C}-\mathrm{C}$ axis at the angles $\alpha$ and $\beta$, respectively. In configuration designated as $\mathrm{C}_{\mathrm{s}} \perp$, the reflection plane is perpendicular to the molecular plane of the $C_{2 v}$ structure.

Many studies of molecules under consideration were devoted to the determination of their equilibrium geometries in the ground and the lowest excited states, vertical excitation energies, potential barriers to inversion, the harmonic frequencies calculations etc. [15-22]. However, the origin of stabilization of their vertically excited states, accompanied by changes in molecular geometry, as well as the reasons of the larger distortion in the triplet state compared with the excited singlet one were not considered at all. 
In all the cases the electronic structure calculations were carried out, the PJTE parameters (non-vibronic contribution to the curvature of the AP, the vibronic coupling constants, the resulting values of the curvature) were estimated, and it was shown that structural distortions of the molecules in the excited states are due to the vibronic mixing of considered states with the appropriate by symmetry higher-lying excited states.

\section{Methods and computational details}

The theory of the PJTE is well developed (See, e.g. in [1-3]). We present here the basic formulas that will be needed in further discussion of the results. In this approach the problem of the stability or instability of molecular nuclear configuration is reduced to the estimation of the curvature $K$ of the adiabatic potential energy surface (APES) of the molecular system in the arbitrary direction $Q_{\Gamma}$ at the high-symmetry configuration $Q_{0}$ for which the first derivatives are zero. The exact expression for $K$ of any molecular system in the state $\left|\Gamma_{1}\right\rangle$, ground or excited, can be obtained in the second order perturbation theory with respect to small nuclear displacements $Q_{\Gamma}$ :

$$
K_{\Gamma}^{\Gamma_{1}}=K_{0 \Gamma}^{\Gamma_{1}}+K_{v \Gamma}^{\Gamma_{1}}=\left\langle\Gamma_{1}\left|\left(\partial^{2} H / \partial Q_{\Gamma}^{2}\right)_{0}\right| \Gamma_{1}\right\rangle-2 \sum_{i} \frac{\left|\left\langle\Gamma_{1}\left|\left(\partial H / \partial Q_{\Gamma}\right)_{0}\right| \Gamma_{i}\right\rangle\right|^{2}}{\left(E_{\Gamma_{i}}-E_{\Gamma_{1}}\right)} .
$$

Here $H$ is the adiabatic electronic Hamiltonian of the system which includes all Coulomb interactions between electrons and nuclei, and $\left|\Gamma_{i}\right\rangle$ and $E_{\Gamma i}$ are the electronic wave functions and the total energies for the equilibrium nuclear configuration, respectively. The first term in Eq.(1), $K_{0 \Gamma}^{\Gamma_{1}}$, is the so-called primary force constant. It determines the restoring force arising when the nuclei are displaced with respect to the "frozen" electronic distribution. The second term, $K_{v \Gamma}^{\Gamma_{1}}$,

$$
K_{v \Gamma}^{\Gamma_{1}}=-2 \sum_{\Gamma i}\left|F_{\Gamma}^{\Gamma_{\Gamma} \Gamma_{i}}\right|^{2} /\left(E_{\Gamma_{i}}-E_{\Gamma_{1}}\right)
$$

results from the vibronic contribution. It takes into account the reduction of $K$ due to the partial "floating" of electrons, their relaxation. This term is always negative if the vibronic mixing takes place only with the higher states. In Eq.(2) $F_{\Gamma}^{\Gamma_{1} \Gamma_{i}}$ is the constant of vibronic coupling between the states $\left|\Gamma_{1}\right\rangle$ and $\left|\Gamma_{i}\right\rangle$ under the distortion $Q_{\Gamma}$ :

$F_{\Gamma}^{\Gamma_{\Gamma} \Gamma_{i}}=\left\langle\Gamma_{1}\left|\left(\partial H / \partial Q_{\Gamma}\right)_{0}\right| \Gamma_{i}\right\rangle$

Note that the vibronic constants $F_{\Gamma}^{\Gamma_{1} \Gamma_{i}}$ and therefore the vibronic contribution $K_{v \Gamma}^{\Gamma_{1}}$ to the curvature of the AP are nonzero only if two states $\Gamma_{1}$ and $\Gamma_{i}$ obey the symmetry restriction: $\Gamma_{1} \otimes \Gamma_{i}$ contains $\Gamma$.

It was proved analytically and confirmed by a series of numerical calculations [4-7] that for any molecular system

$K_{0 \Gamma}^{\Gamma_{1}} \geq 0$

This means that structural instabilities and distortions of high-symmetry nuclear configurations of any polyatomic system in non-degenerate states are only due to the PJTE, i.e. the vibronic mixing of the electronic state under consideration with appropriate higher in energy states under the nuclear displacements in the direction of distortion. The instability takes place if the inequality

$$
\left|K_{v \Gamma}^{\Gamma_{1}}\right| \geq K_{0 \Gamma}^{\Gamma_{1}}
$$

holds, i.e. when the vibronic coupling is strong enough and/or the energy gap between the mixing states is relatively small.

The curvature operator in Eq.(1) contains the part of the Hamiltonian dependent on nuclear coordinates only, i.e. $\partial^{2} H / \partial Q^{2}=\partial^{2} V / \partial Q^{2}$, where

$$
V=-\sum_{\alpha=1}^{N} \sum_{i=1}^{n} \frac{Z_{\alpha}}{\left|\vec{r}_{i}-\vec{R}_{\alpha}\right|}+\frac{1}{2} \sum_{\alpha>\beta}^{N} \frac{Z_{\alpha} Z_{\beta}}{\left|\vec{R}_{\alpha}-\vec{R}_{\beta}\right|}
$$


Here $N$ is the number of atoms, $Z_{\alpha}$ are the nuclear charges, and $\boldsymbol{R}_{\alpha}$ and $\boldsymbol{r}_{i}$ are the nuclear and electron coordinates respectively. It follows that the operator $\partial^{2} H / \partial Q^{2}$ is a sum of one-electron operators, and hence in the one-electron approximation the value of $K_{0}$ equals the sum (over the occupied MOs) of one-electron matrix elements:

$$
K_{0 \Gamma}^{\Gamma_{1}}=\sum_{l}^{o c c} n_{l}\left\langle l\left(\frac{\partial^{2} h(l)}{\partial Q_{\Gamma}^{2}}\right)_{0} \mid l\right\rangle+\frac{\partial^{2}}{\partial Q_{\Gamma}^{2}}\left(\sum_{\alpha>\beta}^{N} \frac{Z_{\alpha} Z_{\beta}}{R_{\alpha \beta}}\right)_{0}
$$

where $n_{l}$ is the occupation number of the $l$-th $\mathrm{MO}$, and $h(l)$ is the one-electron Coulomb operator.

If both the lowest singlet and triplet excited states are formed by one-electron excitation $i \rightarrow \alpha$ from the occupied MO $|i\rangle$ to the unoccupied MO $|\alpha\rangle$, that is the corresponding singlet $\left(\left|S_{i}^{\alpha}\right\rangle\right)$ and triplet $\left(\left|T_{i}^{\alpha}\right\rangle\right)$ wave functions differ from each other only by the spin factor, then $K_{0}\left(\left|S_{i}^{\alpha}\right\rangle\right)=K_{0}\left(\left|T_{i}^{\alpha}\right\rangle\right)$. Hence, the difference in curvature, and therefore in the degree of distortion of the molecule in these excited states is due to the difference in the second term in (1), i.e. in the vibronic contributions to the curvature, $K_{v \Gamma}^{\Gamma_{1}}$. In its turn, the value of the latter depends on the vibronic coupling constant $F_{\Gamma}^{\Gamma_{\Gamma} \Gamma_{i}}$. In the "frozen orbital" approximation the wavefunctions of mixing states $\Gamma_{1}$ and $\Gamma_{i}$ differ from each other by only one spin-orbital $(\alpha \leftrightarrow m)$. In this case the vibronic constants can be calculated as one-electron matrix elements:

$$
F_{\Gamma}^{\Gamma_{1} \Gamma_{i}}=f_{\Gamma}^{(\alpha m)}=\left\langle\varphi_{\alpha}\left|\left(\partial V / \partial Q_{\Gamma}\right)_{0}\right| \varphi_{m}\right\rangle
$$

$\varphi_{\alpha}$ and $\varphi_{m}$ are molecular orbitals. Again, if $\left|S_{1}\right\rangle=\left|S_{i}^{\alpha}\right\rangle$ and $\left|T_{1}\right\rangle=\left|T_{i}^{\alpha}\right\rangle$, and higher states $\left|S_{2}\right\rangle=\left|S_{j}{ }^{\beta}\right\rangle$ and $\left|T_{2}\right\rangle=\left|T_{j}^{\beta}\right\rangle$ are pairwise different from each other only by the spin factor, then from Eq.(8) it follows that

$$
F_{\Gamma}^{S_{1} S_{2}}=F_{\Gamma}^{T_{1} T_{2}}
$$

Therefore, the difference in the vibronic contributions to the curvature of the AP for considered $\left|S_{i}^{\alpha}\right\rangle$ and $\left|T_{i}^{\alpha}\right\rangle$ states depends only on the energy gaps between the mixing states, $\Delta E_{S}=E\left(\left|S_{2}\right\rangle\right)-E\left(\left|S_{1}\right\rangle\right)$ and $\Delta E_{T}=E\left(\left|T_{2}\right\rangle\right)-E\left(\left|T_{1}\right\rangle\right)$.

Thus, to answer the questions whether the system in the reference nuclear configuration is stable or not with respect to any low-symmetry coordinate $Q_{\Gamma}$, the wave functions, energy gaps $\left(E_{\Gamma i}-E_{\Gamma 1}\right)$, and the matrix elements $F_{\Gamma}^{\Gamma_{1} \Gamma_{i}}$ and $K_{0 \Gamma}^{\Gamma_{1}}$ should be calculated for the states that are mixed under the considered displacement. Direct calculation of the vibronic coupling matrix elements involved in the PJT model is rather difficult mathematically. In the present work the numerical values of parameters (primary force constants $K_{0}$ and vibronic coupling constants $F$ ) were estimated by fitting the $a b$ initio data for the APES of the molecules to the general formula, obtained from the vibronic theory. In the case of the two-level problem the AP curves as the functions of $Q_{\Gamma}$ can be expressed as $[1,2]$ :

$\varepsilon_{0}\left(q_{\bar{\Gamma}}\right)=1 / 2 K_{0 \bar{\Gamma}}^{\Gamma} q_{\bar{\Gamma}}^{2}+\Delta / 2-1 / 2 \sqrt{\Delta^{2}+F^{2} q_{\bar{\Gamma}}^{2}}$

and the minima of the APES in this case are given by

$\pm Q_{\min }=\left[\left(F^{2} / K_{0}^{2}\right)-\left(\Delta^{2} / F^{2}\right)\right]^{1 / 2}$,

where $\Delta$ is the energy gap between the mixing states.

Electronic structure calculations of all considered species were performed using the GAMESS quantum chemistry package [23, 24], and the split-valence basis sets 6-31G [25]. The geometry optimization was carried out in the frame of the Hartree-Fock-Roothaan method. The potential energy curves for the molecules in the lowest excited states as the functions of corresponding low-symmetry displacements were calculated taking into consideration configuration interaction with single and double excitations (CISD). The active space of CI included three highest occupied and four lowest unoccupied MOs.

\section{Results and discussion \\ Carbonyl fluoride $\mathrm{F}_{2} \mathrm{CO}$}

In Figure 4 the $\mathrm{MO}$ energy levels scheme and the electronic terms of carbonyl fluoride molecule $\mathrm{F}_{2} \mathrm{CO}$ are presented. It is seen that the lowest singlet and triplet excited states of $\mathrm{A}_{2}$ symmetry are formed by one-electron excitation from the HOMO of $5 b_{2}$ symmetry to the LUMO $3 b_{1}$. Because the out-of-plane distortion, leading to pyramidalization, transforms according to the irreducible representation $\mathrm{B}_{1}$ (Figure 1), then in accordance with the selection rules for the vibronic coupling constants only the excited states of $\mathrm{B}_{2}$ symmetry can mix with the considered $\mathrm{A}_{2}$ states. It is also seen, that the triplet ${ }^{3} \mathrm{~A}_{2}$ and singlet ${ }^{1} \mathrm{~A}_{2}$ states differ from each other only by the spin factor. Hence from Eq. (7) it follows that the value of $K_{0}$ is the same for both singlet and triplet $\mathrm{A}_{2}$ states, $K_{0}^{\mathrm{S}}=K_{0}^{\mathrm{T}}$. The ${ }^{3} \mathrm{~B}_{2}$ and ${ }^{1} \mathrm{~B}_{2}$ states also differ from each other 
only by the spin factor. Then, from the Eq. (9) it follows that $F_{b_{1}}^{A_{2} B_{2}}(S)=F_{b_{1}}^{A_{2} B_{2}}(T)$. Therefore, the difference between the curvatures of the adiabatic potentials for the singlet and triplet excited states, $K^{\mathrm{S}}$ and $K^{\mathrm{T}}$, is due to the difference in the energy gaps between the $\mathrm{A}_{2}$ states and the $\mathrm{B}_{2}$ states, i.e. between the $\Delta E_{T}$ and $\Delta E_{S}$ (Figure $4 b$ ).

a)

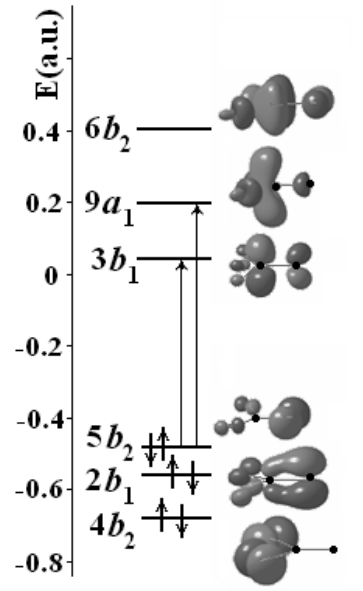

b)

Figure 4. MO energy levels scheme $(a)$ and electronic terms $(b)$ of $\mathrm{F}_{2} \mathrm{CO}$ in the ground state with planar $\left(C_{2 v}\right)$ nuclear configuration. The arrows in $(a)$ indicate the one-electron excitations that form the singlet and triplet $A_{2}$ and $B_{2}$ excited states which are mixed by the vibronic coupling under the $B_{1}$ distortion. In (b) the arrows show tripet-triplet and singlet-singlet vibronic coupled states.

To estimate the values of the PJT parameters according to Eq.(10), the potential energy curves along the outof-plane distortion of $b_{1}$-type were calculated for the triplet excited state of $\mathrm{F}_{2} \mathrm{CO}$ molecule, using two basis sets, 6-31G and tzv (Figure 5). As $Q_{b 1}$, we used the distance from the oxygen atom to the plane $\mathrm{CH}_{2}$. Estimated in such a way values of $K_{0}$ and $F$ are presented in Figure 5.

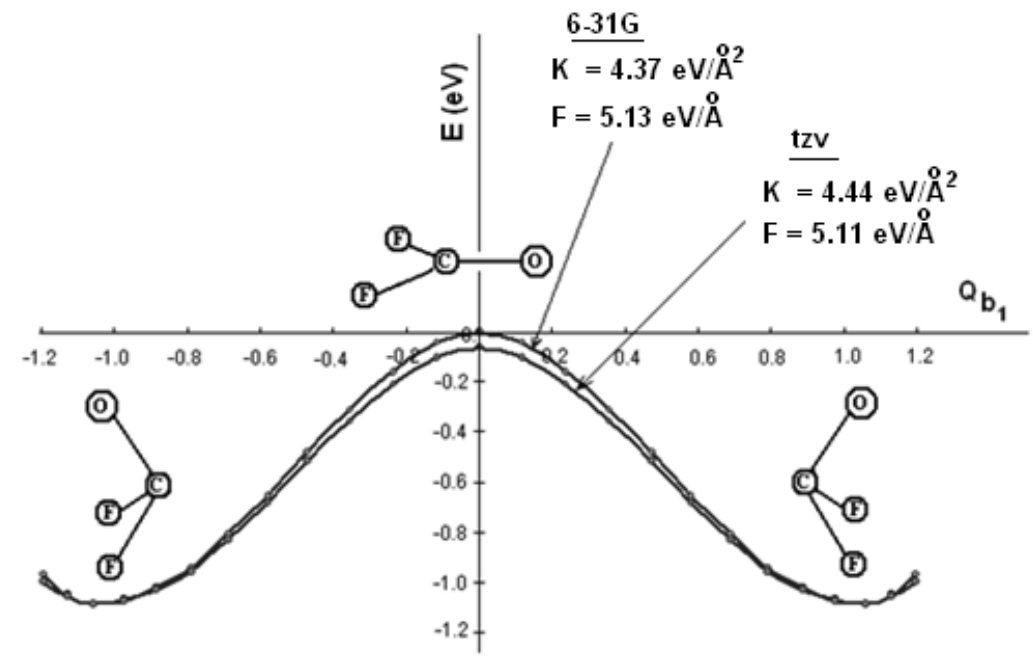

Figure 5. Calculated (marked by points) in the 6-31G and tzv basis sets and analytical (solid lines) AP curves for the ${ }^{3} \mathrm{~A}_{2}$ excited state of $\mathrm{F}_{2} \mathrm{CO}$ along the $Q_{b 1}$ coordinate.

It is seen that only a small difference is observed between these double minimum curves and respectively between the $K_{0}$ and $F$ calculated in different basis sets. Calculated values of the energy gaps are: $\Delta E_{T}=4.92 \mathrm{eV}$ and $\Delta E_{S}$ $=5.34 \mathrm{eV}$. With these parameters, we obtain following result values of curvature of the AP respectively for the triplet and singlet $\mathrm{A}_{2}$ excited states: $K^{\mathrm{T}}=-6.33 \mathrm{eV} / \AA^{2}$ and $K^{\mathrm{S}}=-5.48 \mathrm{eV} / \AA^{2}$. It is seen that the absolute value of the curvature for the triplet state is larger than that for the singlet one: $\left|\mathrm{K}^{\mathrm{T}}\right|>\left|\mathrm{K}^{\mathrm{S}}\right|$. This explains the larger instability and the larger distortion of the triplet excited state as compared with the singlet one. Calculated values of the angles $\alpha_{\mathrm{T}}$ and $\alpha_{\mathrm{S}}$ (Figure 1) for this molecule are: $\angle \alpha_{\mathrm{T}}=52.2^{\circ} \angle \alpha_{\mathrm{S}}=48.7^{\circ}$. 
Diimide $\mathrm{N}_{2} \mathrm{H}_{2}$

The valence electron configuration of the ground state of $\mathrm{N}_{2} \mathrm{H}_{2}$ molecule in the equilibrium trans-planar $\left(\mathrm{C}_{2 \mathrm{n}}\right)$ nuclear configuration is $\left(3 a_{\mathrm{g}}\right)^{2}\left(3 b_{\mathrm{u}}\right)^{2}\left(1 a_{\mathrm{u}}\right)^{2}\left(4 a_{\mathrm{g}}\right)^{2}\left(1 b_{\mathrm{g}}\right)^{0}\left(4 b_{\mathrm{u}}\right)^{0}$ (Figure $\left.6 a\right)$. The scheme of the electronic terms is shown in Figure $6 b$, calculated values of the total and relative energies of the states are given in Table 1.

a)

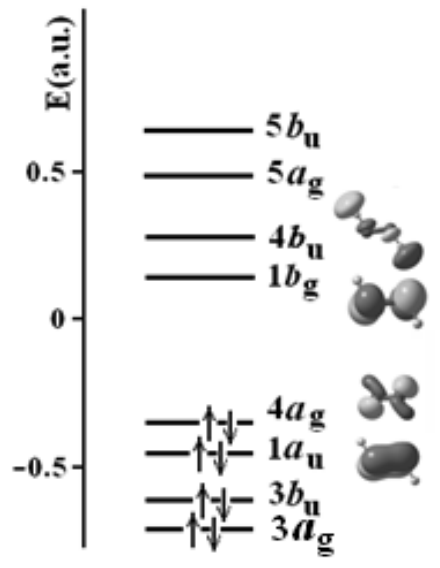

b)

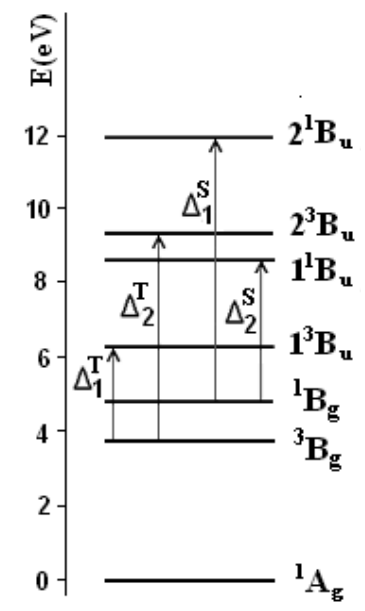

Figure 6. MO energy levels scheme (a) and electronic terms $(b)$ of $\mathrm{N}_{2} \mathrm{H}_{2}$ in the equilibrium trans-planar $\left(\mathrm{C}_{2 \mathrm{~h}}\right)$ nuclear configuration. In $(b)$ the arrows show tripet-triplet and singlet-singlet vibronic coupled states.

Table 1

Total energies (hartree), relative energies (e $V$ ) and electronic configurations of the ground and low-lying excited states for planar $\left(\mathrm{C}_{2 \mathrm{~h}}\right) \mathbf{N}_{\mathbf{2}} \mathbf{H}_{2}$ calculated in tzv basis set.

\begin{tabular}{|c|c|c|c|}
\hline State & Total energy & Relative energy & Electronic configuration \\
\hline${ }^{1} \mathrm{~A}_{\mathrm{g}}$ & -110.01265 & 0 & {$\left[\ldots 3 b_{\mathrm{u}}^{2} 1 a_{\mathrm{u}}^{2} 4 a_{\mathrm{g}}^{2} 1 b_{\mathrm{g}}^{0} 4 b_{\mathrm{u}}^{0}\right]$} \\
\hline${ }^{3} \mathrm{~B}_{\mathrm{g}}$ & -109.88862 & 3.37 & $1 / \sqrt{ } 2\left[\ldots 3 b_{\mathrm{u}}^{2} 1 a_{\mathrm{u}}^{2}\left(4 a_{\mathrm{g}} \overline{1}_{\mathrm{g}}-\overline{4 a}_{\mathrm{g}} 1 b_{\mathrm{g}}\right) 4 b_{\mathrm{u}}^{0}\right]$ \\
\hline${ }^{1} \mathrm{~B}_{\mathrm{g}}$ & -109.84542 & 4.55 & $1 / \sqrt{ } 2\left[\ldots 3 b_{\mathrm{u}}^{2} 1 a_{\mathrm{u}}^{2}\left(4 a_{\mathrm{g}} \overline{1 b}_{\mathrm{g}}+\overline{4 a}_{\mathrm{g}} 1 b_{\mathrm{g}}\right) 4 b_{\mathrm{u}}^{0}\right]$ \\
\hline $1^{3} \mathrm{~B}_{\mathrm{u}}$ & -109.78185 & 6.28 & $1 / \sqrt{ } 2\left[\ldots 3 b_{\mathrm{u}}^{2} 4 a_{\mathrm{g}}^{2}\left(1 a_{\mathrm{u}} \overline{1 b}_{\mathrm{g}}-\overline{1 a}_{\mathrm{u}} 1 b_{\mathrm{g}}\right) 4 b_{\mathrm{u}}^{0}\right]$ \\
\hline $1^{1} \mathrm{~B}_{\mathrm{u}}$ & -109.68997 & 8.78 & $1 / \sqrt{ } 2\left[\ldots 3 b_{\mathrm{u}}^{2} 1 a_{\mathrm{u}}^{2}\left(4 a_{\mathrm{g}} \overline{4 b}_{\mathrm{u}}+\overline{4 a}_{\mathrm{g}} 4 b_{\mathrm{u}}\right) 1 b_{\mathrm{g}}^{0}\right]$ \\
\hline $2^{3} \mathrm{~B}_{\mathrm{u}}$ & -109.65505 & 9.73 & $1 / \sqrt{ } 2\left[\ldots 3 b_{\mathrm{u}}^{2} 1 a_{\mathrm{u}}^{2}\left(4 a_{\mathrm{g}} \overline{4 b}_{\mathrm{u}}-\overline{4 a}_{\mathrm{g}} 4 b_{\mathrm{u}}\right) 1 b_{\mathrm{g}}^{0}\right]$ \\
\hline $2^{1} \mathrm{~B}_{\mathrm{u}}$ & -109.58435 & 11.65 & $1 / \sqrt{ } 2\left[\ldots 3 b_{\mathrm{u}}^{2} 4 a_{\mathrm{g}}^{2}\left(1 a_{\mathrm{u}} \overline{1}_{\mathrm{g}}+\overline{1 a}_{\mathrm{u}} 1 b_{\mathrm{g}}\right) 4 b_{\mathrm{u}}^{0}\right]$ \\
\hline
\end{tabular}

Note that not all obtained excited states are listed in Table 1, but only those that participate in the singletsinglet and triplet-triplet vibronic coupling. From Table 1 and Figure $6 b$ it is seen that the lowest excited singlet and triplet ${ }^{1} \mathrm{~B}_{\mathrm{g}}$ and ${ }^{3} \mathrm{~B}_{\mathrm{g}}$ states are mainly formed by the electron excitation from the nonbonding HOMO $4 a_{\mathrm{g}}\left(\mathrm{n}_{\mathrm{N} 1}-\mathrm{n}_{\mathrm{N} 2}\right)$ to the antibonding LUMO $1 b_{\mathrm{g}}\left(\pi^{*}\right)$ (Figure $6 a$ ). The out-of-plane distortion leading to nonplanar $\mathrm{C}_{2}$ structure is of the $a_{\mathrm{u}}$ type. This means that the direct product of irreducible representations of the two mixing electronic states that contribute to the $a_{\mathrm{u}}$ type instability of the planar configuration must contain the $\mathrm{A}_{\mathrm{u}}$ representation. For the first excited ${ }^{1,3} \mathrm{~B}_{\mathrm{g}}$ states, their mixing with the higher ${ }^{1,3} \mathrm{~B}_{\mathrm{u}}$ states yields the necessary $\mathrm{A}_{\mathrm{u}}$ symmetry: $\mathrm{B}_{\mathrm{g}} \times \mathrm{B}_{\mathrm{u}}=\mathrm{A}_{\mathrm{u}}$. The two such excited states are $1^{1,3} \mathrm{~B}_{\mathrm{u}}$ $\left(1 a_{\mathrm{u}} \rightarrow 1 b_{\mathrm{g}}\right)$ and $2^{1,3} \mathrm{~B}_{\mathrm{u}}\left(4 a_{\mathrm{g}} \rightarrow 4 b_{\mathrm{u}}\right)$. The next $\mathrm{B}_{\mathrm{u}}$ states $\left(4 a_{\mathrm{g}} \rightarrow 5 b_{\mathrm{u}}\right)$ is much higher in energy $(\Delta \mathrm{E}>20 \mathrm{eV})$ and seems to be less important ones.

Since both the excited singlet ${ }^{1} \mathrm{~B}_{\mathrm{g}}$ and triplet ${ }^{3} \mathrm{~B}_{\mathrm{g}}$ states are calculated at the same geometry (of the ground state) and differ from each other only by the spin factor, it means that, as in the previous case, $K_{0}\left({ }^{1} \mathrm{~B}_{\mathrm{g}}\right)=K_{0}\left({ }^{3} \mathrm{~B}_{\mathrm{g}}\right)$. That is, the primary force constant $K_{0}$ in the expression for the curvature $K$ of the AP with respect to the symmetry coordinate $Q_{a \mathrm{u}}$ is the same for both ${ }^{1} \mathrm{~B}_{\mathrm{g}}$ and ${ }^{3} \mathrm{~B}_{\mathrm{g}}$ states. Hence, the difference in curvature, and therefore in the degree of distortion of the molecule in these states is due to the difference in the vibronic contributions to the curvature. From the Eq.(2) it follows:

$$
\begin{aligned}
& K_{v}\left({ }^{1} B_{g}\right)=-2 F_{1}^{2} / \Delta_{1}{ }^{S}-2 F_{2}{ }^{2} / \Delta_{2}{ }^{S}, \\
& K_{v}\left({ }^{3} B_{g}\right)=-2 F_{1}{ }^{2} / \Delta_{1}{ }^{T}-2 F_{2}{ }^{2} / \Delta_{2}{ }^{T},
\end{aligned}
$$


where the constants of the vibronic coupling $F_{1}$ and $F_{2}$ between the singlet-singlet and triplet-triplet excited states are also equal to each other:

$$
\begin{aligned}
& F_{1}=\left\langle{ }^{1} B_{g}\left|\left(\partial H / \partial Q_{a u}\right)_{0}\right| 1^{1} B_{u}\right\rangle=\left\langle{ }^{3} B_{g}\left|\left(\partial H / \partial Q_{a u}\right)_{0}\right| 1^{3} B_{u}\right\rangle \\
& F_{2}=\left\langle{ }^{1} B_{g}\left|\left(\partial H / \partial Q_{a u}\right)_{0}\right| 2{ }^{1} B_{u}\right\rangle=\left\langle{ }^{3} B_{g}\left|\left(\partial H / \partial Q_{a u}\right)_{0}\right| 2^{3} B_{u}\right\rangle
\end{aligned}
$$

Because the mixing ${ }^{1} \mathrm{~B}_{\mathrm{g}}$ and ${ }^{1} \mathrm{~B}_{\mathrm{u}}$ states, as well as the ${ }^{3} \mathrm{~B}_{\mathrm{g}}$ and ${ }^{3} \mathrm{~B}_{\mathrm{u}}$ states differ from each other by only one spin-orbital, then in accordance with Eq.(8) we obtain:

$$
\begin{aligned}
& F_{1}=f_{1}=\left\langle 1 a_{u}\left|\left(\partial H / \partial Q_{a u}\right)_{0}\right| 4 a_{g}\right\rangle, \\
& F_{2}=f_{2}=\left\langle 1 b_{g}\left|\left(\partial H / \partial Q_{a u}\right)_{0}\right| 4 b_{u}\right\rangle,
\end{aligned}
$$

and for $K_{v}$ we can write: $K_{v}\left({ }^{1} B_{g}\right)=-2 f_{1}^{2} / \Delta_{1}{ }^{S}-2 f_{2}^{2} / \Delta_{2}{ }^{S}$, and $K_{v}\left({ }^{3} B_{g}\right)=-2 f_{1}^{2} / \Delta_{1}{ }^{T}-2 f_{2}^{2} / \Delta_{2}{ }^{T}$.

To estimate the values of the orbital vibronic coupling constants $f_{1}$ and $f_{2}$ the following procedure is applied. From the $a b$ initio calculations the curvature of the APES with respect to the $a$ distortion is determined for the transplanar nuclear configurations of $\mathrm{C}_{2 \mathrm{~h}}$ symmetry of the neutral $\mathrm{N}_{2} \mathrm{H}_{2}$ molecule, of the anion $\mathrm{N}_{2} \mathrm{H}_{2}^{-}$and the cation $\mathrm{N}_{2} \mathrm{H}_{2}^{+}$. Calculated values are: $K\left(\mathrm{~N}_{2} \mathrm{H}_{2}\right)=12.60 \mathrm{eV} / \AA^{2}$ (the experimental value $K=\mathrm{M} \omega^{2}$ with $\omega\left(a_{\mathrm{u}}\right)=1290 \mathrm{~cm}^{-1}$ [26] is 11.52 $\left.\mathrm{eV} / \AA^{2}\right), K\left(\mathrm{~N}_{2} \mathrm{H}_{2}^{+}\right)=5.78 \mathrm{eV} / \AA^{2}$, and $K\left(\mathrm{~N}_{2} \mathrm{H}_{2}^{-}\right)=3.10 \mathrm{eV} / \AA^{2}$. The softening of the $\mathrm{N}_{2} \mathrm{H}_{2}^{+}$with respect to the $a_{\mathrm{u}}$ distortion is due to the vibronic coupling between the double occupied $1 a_{\mathrm{u}} \mathrm{MO}$ and single occupied $4 a_{\mathrm{g}} \mathrm{MO}, \Delta K=f_{1}^{2} / \Delta E_{\text {cation }}$, $\Delta E_{\text {cation }}=\mathrm{E}\left({ }^{2} \mathrm{~A}_{\mathrm{u}}\right)-\mathrm{E}\left({ }^{2} \mathrm{~A}_{\mathrm{g}}\right)$, while the softening of the $\mathrm{N}_{2} \mathrm{H}_{2}-$ is due to the vibronic coupling between the single occupied $1 b_{\mathrm{g}}$ MO and vacant $4 b_{\mathrm{u}} \mathrm{MO}, \Delta K=f_{2}^{2} / \Delta E_{\text {anion, }} \Delta E_{\text {anion }}=\mathrm{E}\left({ }^{2} \mathrm{~B}_{\mathrm{u}}\right)-\mathrm{E}\left({ }^{2} \mathrm{~B}_{\mathrm{g}}\right)$. Thus, we obtain the following values for the vibronic coupling constants in the $\mathrm{N}_{2} \mathrm{H}_{2}$ molecule: $f_{1}=4.25 \mathrm{eV} / \AA$ and $f_{2}=4.73 \mathrm{eV} / \AA$.

Using the values of $\Delta E_{i}^{S, T}$ from Table 1 , we obtain $K_{\mathrm{v}}\left({ }^{1} \mathrm{~B}_{\mathrm{g}}\right)=-15.67 \mathrm{eV} / \AA^{2}$ and $K_{\mathrm{v}}\left({ }^{3} \mathrm{~B}_{\mathrm{g}}\right)=-19.45 \mathrm{eV} / \AA^{2}$. As the $K_{0}$ we used the value of $K$ for the ground state because the latter is not mixed with any excited states of $\mathrm{A}_{\mathrm{u}}$ symmetry. Finally we get $K\left({ }^{1} \mathrm{~B}_{\mathrm{g}}\right)=-3.07 \mathrm{eV} / \AA^{2}$ and $K\left({ }^{3} \mathrm{~B}_{\mathrm{g}}\right)=-6.85 \mathrm{eV} / \AA^{2}$. Calculations point-by-point give the following values of $K$ in the neighborhood of $Q_{a u}=0: K\left({ }^{1} \mathrm{~B}_{\mathrm{g}}\right)=-3.20 \mathrm{eV} / \AA^{2}$ and $K\left({ }^{3} \mathrm{~B}_{\mathrm{g}}\right)=-6.48 \mathrm{eV} / \AA^{2}$. Thus, in this case we also obtain $\left|\mathrm{K}^{\mathrm{T}}\right|>\left|\mathrm{K}^{\mathrm{S}}\right|$. That is why the distortion of $\mathrm{N}_{2} \mathrm{H}_{2}$ in the triplet state is stronger than that in the singlet one. Calculated values of the dihedral angles $\alpha_{\mathrm{T}}$ and $\alpha_{\mathrm{S}}$ (Figure 2) for this molecule are: $\leq \alpha_{\mathrm{T}}=95.5^{\circ}$ and $\leq \alpha_{\mathrm{S}}=106.2^{\circ}$, and the pseudo Jahn-Teller stabilization energies are equal to $\Delta E_{P J T}^{T}=0.67 \mathrm{eV}$ and $\Delta E_{P J T}^{S}=0.25 \mathrm{eV}$.

\section{Ketene $\mathrm{H}_{2} \mathrm{C}_{2} \mathrm{O}$}

The five-atom ketene molecule $\left(\mathrm{H}_{2} \mathrm{CCO}\right)$ has nine vibrational degrees of freedom. In the planar configuration of $\mathrm{C}_{2 \mathrm{v}}$ symmetry (configuration of the ground electronic state) they transform according to the irreducible representations $4 a_{1}+2 b_{1}+3 b_{2}$. Three symmetrized displacements of the $b_{2}$-type (the $\mathrm{C}$-C-O in-plane bending, the $\mathrm{CH}_{2}$ fragment in-plane wagging, and the $\mathrm{C}-\mathrm{H}$ asymmetric stretch) reduce the symmetry from $\mathrm{C}_{2 \mathrm{v}}$ to $\mathrm{C}_{\mathrm{S}}$ and lead to the $\mathrm{C}_{\mathrm{S}}$ " structure (Figure 3 ). Two low-symmetry $b_{1}$ modes (the $\mathrm{C}-\mathrm{C}$-O out-of-plane bending and the $\mathrm{CH}_{2}$ fragment out-of-plane wagging) correspond to the out-of-plane bending of the molecule and transform the planar nuclear configuration into the $\mathrm{C}_{\mathrm{S}}{ }^{\perp}$ one (Figure 3 ). Calculated parameters of the geometrical structure in the ground $\left({ }^{1} \mathrm{~A}_{1}\right)$ and excited $\left({ }^{3} \mathrm{~A}^{\prime \prime}\right.$ and $\left.{ }^{3} \mathrm{~A}^{\prime}\right)$ states of ketene are presented in Table 2. They agree rather well with those of other authors [21, 22].

Table 2

Optimized geometry parameters in the ground $\left({ }^{1} \mathrm{~A}_{1}\right)$ and excited $\left({ }^{3} \mathrm{~A}^{\prime}\right.$ and $\left.{ }^{3} \mathrm{~A}^{\prime}\right)$ states of ketene $\mathrm{e}^{\mathrm{a}}$

\begin{tabular}{cccccccccc}
\hline Symmetry & State & $R_{C-O}$ & $R_{C-C}$ & $R_{C-H}$ & $\angle H C H$ & $\alpha$ & $\beta$ & $\gamma$ & $\delta$ \\
\hline $\mathrm{C}_{2 \mathbf{v}}$ & ${ }^{1} \mathrm{~A}_{1}$ & 1.167 & 1.303 & 1.069 & 119.98 & - & - & - \\
$\mathrm{C}_{\mathrm{S}}$ & ${ }^{3} \mathrm{~A}^{\prime}\left({ }^{3} \mathrm{~A}_{1}\right)$ & 1.191 & 1.482 & 1.072 & 122.38 & - & - & 55.3 & 22.7 \\
$\mathrm{C}_{\mathrm{S}}{ }^{I I}$ & ${ }^{3} \mathrm{~A}^{\prime \prime}\left({ }^{3} \mathrm{~A}_{2}\right)$ & 1.193 & 1.449 & $1.073\left(\mathrm{C}-\mathrm{H}_{1}\right)$ & 119.81 & 49.3 & 120.36 & - & - \\
& & & & & & & & & \\
\end{tabular}

${ }^{a}$ Bond lengths are in $\AA$, angles are in degrees; angles $\alpha, \beta, \gamma$ and $\delta$ are shown in Figure 3 .

The MO energy level scheme and the electronic terms of ketene molecule in the equilibrium planar $\left(\mathrm{C}_{2 \mathrm{v}}\right)$ nuclear configuration are presented in Figure 7. We see that the first excited ${ }^{3} \mathrm{~A}_{2}$ state is formed by the electron excitation from the HOMO $2 b_{1}$ which is the $\mathrm{C}-\mathrm{C}$ bonding one, to the LUMO $3 b_{2}$, the antibonding MO with respect to $\mathrm{C}-\mathrm{O}$ bond. Because the in-plane distortion is of the $b_{2}$ type, then only the higher excited ${ }^{3} \mathrm{~B}_{1}$ state contributes to the $b_{2}$ type instability of the $\mathrm{C}_{2 \mathrm{v}}$ configuration: $\mathrm{A}_{2} \times \mathrm{B}_{1}=\mathrm{B}_{2}$. 
(a)

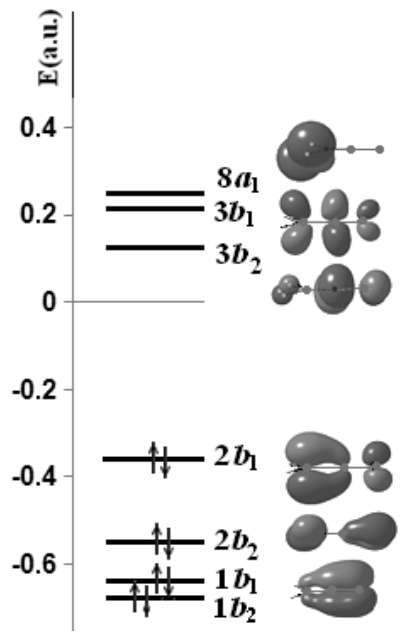

(b)

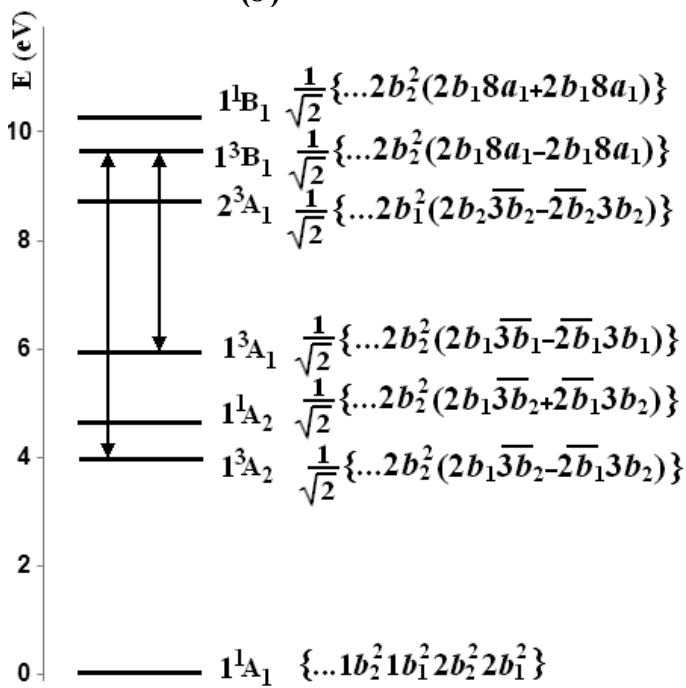

Figure 7. MO energy levels scheme (a) and electronic terms (b) of ketene molecule in the ground state with planar $\left(C_{2 v}\right)$ nuclear configuration. The arrows indicate the vibronic coupled excited states.

The ${ }^{3} \mathrm{~A}_{1}$ state is formed by the one-electron excitation $2 b_{1} \rightarrow 3 b_{1}$, the latter is the antibonding MO for both the C-C and $\mathrm{C}-\mathrm{O}$ bonds. In this case the vibronic contribution to the curvature of the AP along the out-of-plane distortion of the $b_{1}$ type is determined by the PJT mixing also with the ${ }^{3} \mathrm{~B}_{1}$ excited state: $\mathrm{A}_{1} \times \mathrm{B}_{1}=\mathrm{B}_{1}$.

Generally speaking, in order to reveal the vibronic origin of the in-plane distortion of ketene molecule in the ${ }^{3} \mathrm{~A}_{2}$ state we must solve the three-mode $\left(\mathrm{A}_{2}+\mathrm{B}_{1}\right) \otimes\left(b_{2}+b_{2}+b_{2}\right)$ pseudo Jahn-Teller problem. For the out-of-plane distortion the two-mode $\left(\mathrm{A}_{1}+\mathrm{B}_{1}\right) \otimes\left(b_{1}+b_{1}\right)$ problem arises. Then the resulting normal coordinates $Q_{b 2}$ and $Q_{b 1}$ of the $b_{2}$-type and $b_{1}$-type displacements are obtained as the eigenvectors of the matrices $K_{i j}(i=1,2,3$ for the three symmetrized displacements of the $b_{2}$-type, and $i=1,2$ for those of the $b_{1}$-type)

$$
K_{i j}=K_{i j}^{(0)}-2 \frac{F^{(i)} F^{(j)}}{E\left({ }^{3} B_{1}\right)-E\left({ }^{3} A_{2}\left(o r^{3} A_{1}\right)\right)},
$$

corresponding to the negative eigenvalues of the latest. Fortunately, the problem can be simplified taking into account that the $Q_{b 2}$ and $Q_{b 1}$ coordinates coincides with the line of steepest decrease of the energy in the space of symmetrized coordinates that connects the $\mathrm{C}_{2 \mathrm{v}}$ and $\mathrm{C}_{\mathrm{S}}{ }^{\mathrm{II}}\left(\mathrm{C}_{\mathrm{S}}{ }^{\perp}\right)$ minima. From Table 2 one can see that the $Q_{h 2}$ is determined almost exclusively by the in-plane $\mathrm{C}-\mathrm{C}-\mathrm{O}$ bending, the $\mathrm{CH}_{2}$ fragment in-plane wagging and the $\mathrm{C}-\mathrm{H}$ asymmetric stretch are negligibly small. That is why in further calculations of the AP curve along the in-plane distortion we used as the $Q_{b 2}$ the deviation of the oxygen atom from the $\mathrm{C}-\mathrm{C}$ axis. Calculations of the AP curve along the out-of-plane distortion showed that the $\mathrm{CH}_{2}$ fragment out-of-plane wagging (change of the angle $\delta$ in Figure 3 ) has little effect on the value of the total energy (about $0.02 \mathrm{eV}$ ). Therefore, as $Q_{b 1}$ we used the out-of-plane displacement of the oxygen atom.

Calculated adiabatic potential curves as the functions of $Q_{b 2}$ for the first triplet state of ketene, and of $Q_{b 1}$ for the second excited triplet state are shown in Figure 8. As in the case of carbonyl fluoride we evaluated parameters $K_{0}$ and $F$ by fitting the ab initio data for the AP curves to the general formula from Eq.(10). Estimated in such a way values of the PJT parameters are presented in Figure 8, where

$$
F\left(b_{2}\right)=F_{b 2}^{A 2, B 1}=\left\langle{ }^{3} A_{2}\left|\left(\partial H / \partial Q_{b 2}\right)_{0}\right|{ }^{3} B_{1}\right\rangle \text { and } F\left(b_{1}\right)=F_{b 1}^{A 1, B 1}=\left\langle{ }^{3} A_{1}\left|\left(\partial H / \partial Q_{b 1}\right)_{0}\right|^{3} B_{1}\right\rangle .
$$

From Figure 8 it is seen that calculated potential energy curves for the ${ }^{3} \mathrm{~A}_{2}(a)$ and ${ }^{3} \mathrm{~A}_{1}(b)$ excited states along the corresponding low-symmetry distortions ( $b_{2}$-type in the first case and $b_{1}$-type in the second one) are fully coincide with those predicted from the general theory. Thus, we can conclude that the instability of high-symmetry $\mathrm{C}_{2 \mathrm{v}}$ nuclear configuration of ketene molecule in considered ${ }^{3} \mathrm{~A}_{2}$ and ${ }^{3} \mathrm{~A}_{1}$ excited states and the corresponding low-symmetry distortions are due to the pseudo Jahn-Teller mixing of these states with the higher excited ${ }^{3} \mathrm{~B}_{1}$ state. 
(a)

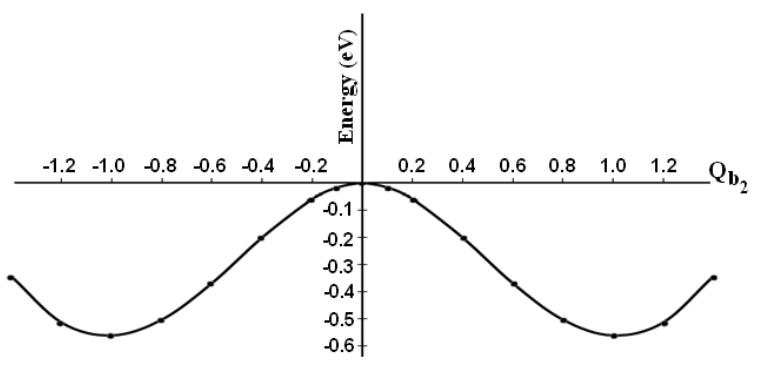

$$
\begin{gathered}
\left({ }^{3} \mathbf{A}_{2}+{ }^{3} \mathbf{B}_{1}\right) \otimes \boldsymbol{b}_{2}: \\
K_{0}=5.81 \mathrm{eV} / \AA^{2} \\
F\left(b_{2}\right)=6.16 \mathrm{eV} / \AA \\
\Delta=5.53 \mathrm{eV}
\end{gathered}
$$$$
K_{b 2}\left({ }^{3} \mathrm{~A}_{2}\right)=-7.91 \mathrm{eV} / \AA^{2}
$$

(b)

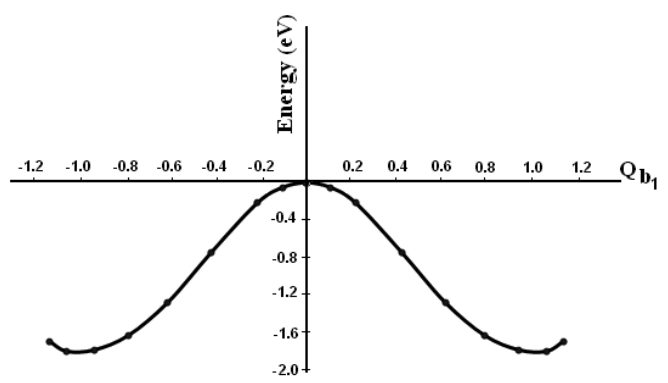

$$
\begin{gathered}
\left({ }^{3} \mathbf{A}_{\mathbf{1}}+{ }^{3} \mathbf{B}_{\mathbf{B}}\right) \otimes \boldsymbol{b}_{\mathbf{1}}: \\
K_{0}=5.19 \mathrm{eV} / \AA^{2} \\
F\left(b_{1}\right)=5.47 \mathrm{eV} / \AA \\
\Delta=3.56 \mathrm{eV}
\end{gathered}
$$

Figure 8. Calculated (marked by points) and analytical (solid lines) AP curves for the ${ }^{3} \mathrm{~A}_{2}(a)$ and ${ }^{3} \mathrm{~A}_{1}(b)$ excited states along the $Q_{b 2}$ and $Q_{b 1}$ coordinates.

\section{Diagonal vibronic constants for the excited states}

In the same approximation, by applying the above equations to the molecules with respect to the totally symmetric displacements, one can explain also the elongation of $\mathrm{C}-\mathrm{O}, \mathrm{N}-\mathrm{N}$, and $\mathrm{C}-\mathrm{C}$ bonds in excited states of $\mathrm{F}_{2} \mathrm{CO}$, $\mathrm{N}_{2} \mathrm{H}_{2}$, and $\mathrm{H}_{2} \mathrm{C}_{2} \mathrm{O}$ molecules, respectively. These distortions do not change the symmetry of the molecules, but change the interatomic distances. The diagonal vibronic coupling constant $F_{Q}^{\Gamma \Gamma}=\left\langle\Gamma\left|(\partial H / \partial Q)_{0}\right| \Gamma\right\rangle$ has the physical meaning of the force with which the electrons in the state $|\Gamma\rangle$ affect the nuclei in the direction of $Q$ [1, p. 340]. In the one-electron approximation this constant equals the sum of diagonal orbital vibronic coupling constants (OVCC) multiplied by the appropriate $\mathrm{MO}$ occupation numbers, $n_{l}$ :

$F_{Q}=\sum_{l}^{o c c} n_{l} f_{Q}^{l l}$

where

$$
f_{Q}^{l l}=\left\langle l\left|(\partial V / \partial Q)_{0}\right| l\right\rangle
$$

are the linear diagonal orbital vibronic constants. These values can be both positive and negative, depending on the character of the MO: for a bonding (relative to the considered bond) molecular orbital $f_{Q}^{l l}>0$, for an antibonding MO $f_{Q}^{l l}<0$, and for nonbonding $\mathrm{MO} f_{Q}^{l l} \cong 0 \quad$ [1, p. 342]. In the ground state equilibrium configuration $F_{Q}^{\Gamma \Gamma}$ equals zero. However, any change of the electron structure of the molecule results in a nonzero distorting force $\Delta F_{Q} \neq 0$. If the excited state is formed by the one-electron excitation $i \rightarrow \alpha$, then

$$
\Delta F_{Q}=f_{Q}^{\alpha \alpha}-f_{Q}^{i i}
$$

In our cases, as shown above, the lowest excited states are formed by the one-electron excitation from the nonbonding $\left(\mathrm{F}_{2} \mathrm{CO}, \mathrm{N}_{2} \mathrm{H}_{2}\right)$ or the bonding $\left(\mathrm{H}_{2} \mathrm{C}_{2} \mathrm{O}\right) \mathrm{MOs}$ to the antibonding MOs of corresponding molecules. Hence, $f_{Q}^{i i} \geq 0, f_{Q}^{\alpha \alpha}<0$, and we get that in all the cases $\Delta F_{Q}<0$. Thus, for all the considered molecules their excitation induces a JTE distorting force which pushes away the nuclei and thereby increases the C-O, N-N, and C-C bond lengths.

\section{Conclusions}

From the results of this paper the following conclusions emerge.

The nonplanar geometry of carbonyl fluoride and diazene molecules in the lowest singlet and triplet excited states, as well as the in-plane and the out-of-plane distortions of ketene molecule in the first and the second triplet states respectively, are due to the pseudo Jahn-Teller mixing of these states with the higher excited states of appropriate 
symmetry. This vibronic coupling produces the low-symmetry distortion of the planar nuclear configurations, as predicted by the general theory.

The elongation of $\mathrm{C}-\mathrm{O}, \mathrm{N}-\mathrm{N}$, and $\mathrm{C}-\mathrm{C}$ bonds respectively in excited states of $\mathrm{F}_{2} \mathrm{CO}, \mathrm{N}_{2} \mathrm{H}_{2}$, and $\mathrm{H}_{2} \mathrm{C}_{2} \mathrm{O}$ molecules, as compared with the bond lengths in their ground states is due to the change of the corresponding diagonal vibronic coupling constants induced by the electronic excitation.

\section{References}

1. Bersuker, I.B. Electronic Structure and Properties of Transition Metal Compounds. Introduction to the Theory, Second Ed.; Wiley: New York, 2010, 759 p.

2. Bersuker, I.B. The Jahn-Teller Effect; Cambridge University Press: Cambridge (UK), 2006, 616 p.

3. Bersuker, I.B.; Polinger, V. Z., Vibronic Interactions in Molecules and Crystals. Springer: New York, 1989,422 p.

4. Bersuker, I.B. Symmetry, instability, stereochemistry and phase transitions in coordination compounds. Pure and Applied Chemistry, 1988, 60, pp. 1167-1174.

5. Bersuker, I. B. Proof of the vibronic origin of an instability in structural phase transitions occurring in condensed media. Soviet Physics. Solid State, 1988, 30, pp. 1000-1003.

6. Bersuker, I.B.; Gorinchoi, N.N.; Polinger, V.Z. On the origin of dynamic instability of molecular systems. Theoretica Chimica Acta, 1984, 66, pp. 161-172.

7. Bersuker, I.B.; Polinger, V.Z.; Gorinchoi, N.N. Pseudo Jahn-Teller origin of square-planar configuration instability of main-groul-element hydrides. Journal of Molecular Structure: THEOCHEM, 1992, 270, pp. 369-380.

8. Reinen, D.; Atanasov, M. Fluxionality and stereochemistry of 5-coordinated $\mathrm{Cu}^{2+}$ complexes. Chemical Physics, 1989, 136, pp. 27-46.

9. Reinen D.; Atanasov M. Symmetry and vibronic coupling: The stereochemistry and the ground state potential surface of $\mathrm{Cu}^{2+}$ in five-coordination. Chemical Physics, 1991, 155, pp. 157-171.

10. Gorinchoi, N.N.; Bersuker, I.B.; Polinger, V.Z. Electronic control of molecular configuration instability via vibronic coupling. Comparison of $\mathrm{CuCl}_{5}{ }^{3+}$ with $\mathrm{ZnCl}_{5}{ }^{3+}$. New Journal of Chemistry, 1993, 17, pp. 125-130.

11. Polinger, V.Z.; Gorinchoi, N.N.; Bersuker, I.B. MO LCAO analysis of the vibronic instability of the $\mathrm{CuCl}_{5}^{3-}$ trigonal bipyramidal configuration. Critical view on the angular overlap model in vibronic problems. Chemical Physics, 1992, 159, pp. 75-87.

12. Gorinchoy, N.N.; Ogurtsov, I.Ya.; Arsene, I. Vibronic origin of the "skewed" anticline configuration of the hydrogen peroxide molecule. Chemistry Journal of Moldova, 2008, 3, pp. 105-111.

13. Ogurtsov, I.; Gorinchoy, N.; Balan, I. Vibronic origin of the $\mathrm{H}_{3} \mathrm{O}$ metastability. Journal of Molecular Structure., 2007, 838, pp. 107-111.

14. Ogurtsov, I.Ya.; Munteanu, G.; Bantush, L.; Bersuker, I.B. Pseudo Jahn-Teller origin of formaldehyde molecular in excited states. Journal of Molecular Structure: THEOCHEM, 2001, 541, pp. 141-148.

15. Choi, H.; Baeck, K. K. Theoretical study of low-lying excited states of $\mathrm{X}_{2} \mathrm{CY}(\mathrm{X}=\mathrm{F}, \mathrm{Cl} ; \mathrm{Y}=\mathrm{O}, \mathrm{S})$ using the equationof-motion coupled-cluster theory. Molecular Physics, 2005, 103, pp. 2247-2254.

16. Godunov, I.A.; Abramenkov, A.V.; Bataev, V.A.; Pupyshev, V.I. Potential functions of inversion of $\mathrm{R}_{2} \mathrm{CO}$ molecules in the lowest excited electronic states. Russian Chemical Bulletin, 1999, 48, pp. 640-646.

17. Bokarev, S.I.; Dolgov, E.K.; Bataev, V.A.; Godunov, I.A. Molecular parameters of tetraatomic carbonyls $\mathrm{X}_{2} \mathrm{CO}$ and $\mathrm{XYCO}(\mathrm{X}, \mathrm{Y}=\mathrm{H}, \mathrm{F}, \mathrm{Cl})$ in the ground and lowest excited electronic states, Part 1: a test of ab initio methods. International Journal of Quantum Chemistry, 2009, 109, pp. 569-585.

18. Kim, K.; Shavitt, I.; Del Bene, J.E. Theoretical study of the di-imide $\left(\mathrm{N}_{2} \mathrm{H}_{2}\right)$ molecule in ground and $\mathrm{n} \rightarrow \pi^{*}$ excited states. Journal of Chemical Physics, 1992, 96, pp. 7573-7579.

19. Tavernelli, I.; Röhrig, U.F.; Rothlisberger, U. Molecular dynamics in electronically excited states using timedependent density functional theory. Molecular Physics, 2005,103, pp. 963-981.

20. Dhont, G.S.F.; van Lenthe, J.H.; Groenenboom, G.C.; van der Avoird, Ad. Ab initio calculation of the $\left.\mathrm{NH}^{3} \Sigma^{-}\right)$$\mathrm{NH}\left({ }^{3} \Sigma^{-}\right)$interaction potentials in the quintet, triplet, and singlet states. Journal of Chemical Physics, 2005, 123, 184302.

21. Dykstra, C.E.; Schaefer III, H.F. Excited electronic states of ketene. Journal of American Chemical Society 1976 , 98, pp. 2689-2695.

22. Szalay, P.G.; Császár, A.G.; Nemes, L. Electronic states of ketene. Journal of Chemical Physics, 1996, 105, pp. 1034-1044.

23. Granovsky, A.A. http://classic.chem.msu.su/gran/gamess/index.html.

24. Schmidt, M.W. et al. General atomic and molecular electronic structure system. Journal Computational Chemistry, 1993, 14, pp. 1347-1363.

25. Hehre, W.J.; Ditchfield, R.; Pople, J.A. Self-Consistent Molecular Orbital Methods. XII. Further Extensions of Gaussian-Type Basis Sets for Use in Molecular Orbital Studies of Organic Molecules. Journal of Chemical Physics, 1972, 56, 2257.

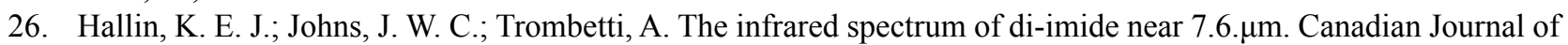
Physics, 1981, 59, pp. 663-672. 